\title{
FERMILAB-CONF-05-239-AD SIMULTANEOUS POSITION MEASUREMENTS OF PROTONS AND ANTI-PROTONS IN THE TEVATRON*
}

\begin{abstract}
R. K. Kutschke ${ }^{\dagger}$, J. Steimel, R. Webber,
Abstract

Fermilab is nearing completion of an upgrade to the electronics of the Beam Position Monitor (BPM) system that measures the transverse position of the beams inside the Tevatron collider. A new feature in the upgraded system is the ability, when both protons and anti-protons are present in the Tevatron, to make simultaneous measurements of the closed orbit position of both beam species. This paper will present one of the methods for achieving the simultaneous measurement and will present results from commissioning data, which demonstrate that the system achieves its requirements.
\end{abstract}

\section{INTRODUCTION}

The stripline directional-coupler design of the Tevatron BPM pickups[1] would ideally offer perfect isolation between signals from particles traveling in opposite directions. In reality, little more than $26 \mathrm{~dB}$ isolation is available at the $53 \mathrm{MHz}$ processing frequency. With the now-typical 6:1 proton-to-antiproton bunch intensity ratio, this isolation alone is insufficient to support millimeter-accuracy antiproton $(\bar{p})$ position measurements in the presence of protons $(p)$. An accurate and manageable solution to this interfering signal problem is required for $\bar{p}$ measurements now and, as $\bar{p}$ intensity increases, to facilitate elimination of $\bar{p}$ bias on $p$ measurements in the future. Two avenues of approach are suggested: 1$)$ separate the signals in the time domain, and 2) calibrate the cross-talk in the frequency domain and make compensation before computing beam position. This paper discusses the second approach; the first is discussed elsewhere [2]. An overview of the BPM upgrade project has has also been contributed to this conference [3].

Two of the key $\bar{p}$ requirements for the upgraded system are an absolute accuracy of $<1 \mathrm{~mm}$ and best orbit position resolution of $<50 \mu \mathrm{m}(1 \sigma)$. This paper will show $\bar{p}$ accuracy measurements relative to measured $p$ position.

\section{METHODOLOGY}

Each BPM station consists of two stripline electrodes, referred to as A and B, each of which is read out at both ends, referred to as the $p$ and $\bar{p}$ ends. If the pickups were perfectly direction-coupled, the signals from each beam species would pass $100 \%$ into the end named after it. The four signals from each BPM station are passed through a band-pass filter, centered at $53 \mathrm{MHz}$, and into an Echotek

\footnotetext{
${ }^{*}$ Fermilab is operated by Universities Research Association Inc. under Contract No. DE-AC02-76CH03000 with the United States Department of Energy.

${ }^{\dagger}$ kutschke@fnal.gov
}

digital receiver board, which is programmed to measure the Fourier amplitude of each signal in a narrow frequency band around $53 \mathrm{MHz}$. A single raw measurement produced by this system consists of 4 complex numbers, $A_{p}, B_{p}, A_{\bar{p}}$ and $B_{\bar{p}}$. Further details of the signal processing may be found elsewhere [4].

In collider operation, the Tevatron beam consists of 36 bunches each of counter-circulating $p$ 's and $\vec{p}$ 's within the common beam tube. For the measurements discussed here, the digital receiver board is programmed in closed orbit mode; that is, it integrates over approximately 50 turns of the Tevatron, which corresponds to a resolution bandwidth of about $1 \mathrm{kHz}$. This measurement is averaged over all of the bunches in the machine and over many turns of each bunch. The integration time is sufficiently long to average out the betatron oscillations but not the synchrotron oscillations. Moreover, the long integration time ensures that the method requires only coarse timing, $\mathrm{O}(100 \mathrm{~ns})$, and the narrow resolution bandwidth reduces the dependence of the position measurement on bunch shape.

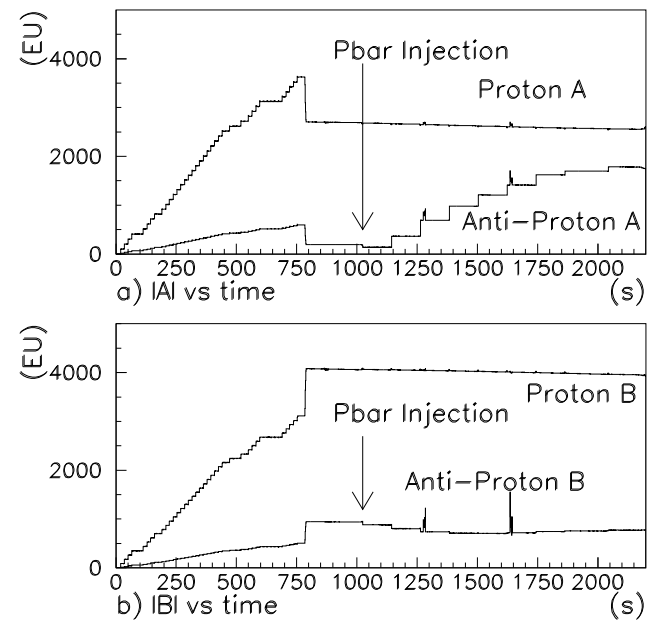

Figure 1: Magnitudes of the raw signals on the four channels from the BPM HB34. The time axis is in seconds from the start of the data set. The vertical axis is in Echotek units.

Figure 1 shows the magnitudes of the signals from each of the four channels on one BPM for the first 36 minutes of a Tevatron shot. On the $\left|A_{p}\right|$ and $\left|B_{p}\right|$ traces, one can see the 36 steps corresponding to the injection of $36 p$ bunches. These bunches are injected onto the central orbit. At about $800 \mathrm{~s}$ the separators are energized, moving the beam onto the $p$ helix and giving rise to steps in $\left|A_{p}\right|$ and $\left|B_{p}\right|$. The vertical arrows mark the beginning of the $\bar{p}$ injection. The $\left|A_{\bar{p}}\right|$ and $\left|B_{\bar{p}}\right|$ traces to the left of the arrow show that the $p$ contamination on the $\bar{p}$ channels is significant. The two 
jumps in the traces, near 1300 and $1600 \mathrm{~s}$, occur when the $\bar{p}$ bunches are cogged relative to the $p$ bunches.

Until the Tevatron $\bar{p}$ currents are increased significantly the $p$ raw measurements can be used without correction. The $\bar{p}$ raw measurements, on the other hand, need to be corrected and studies have shown that a linear model meets the requirements:

$$
\begin{gathered}
A_{\bar{p}}^{\prime}=A_{\bar{p}}-a A_{p}-b B_{p} \\
B_{\bar{p}}^{\prime}=B_{\bar{p}}-c B_{p}-d A_{p},
\end{gathered}
$$

where the primed quantities are the corrected ones and where $a, b, c, d$ are complex parameters, referred to as cancellation coefficients. To determine these coefficients two sets of raw measurements are taken, one at a time, $t_{1}$, just before the helix opens and another at a time $t_{2}$, a few seconds later, just after the helix opens. At both of these times there are no antiprotons in the Tevatron so the corrected antiproton signal at both times should be zero. Using the two raw measurements and setting the left hand side of equation 1 to zero, one can solve for the cancellation coefficients one can solve for the cancellation coefficients. In the following, all references to an unadorned $A$ or $B$ should be interpreted as raw if referring to $p$ signals and as corrected if referring to $\bar{p}$ signals.

\section{RESULTS}

Figure 2a) shows the quantity $|A|+|B|$, referred to as the sum signal, for both beam species for the first hour of a shot. For a constant beam energy, $|A|+|B|$ is proportional to the beam intensity. Both traces show a rise in the sum signal at a time of about $2200 \mathrm{~s}$, which is an artifact due to the ramping of the Tevatron energy from $150 \mathrm{GeV}$ to $980 \mathrm{GeV}$. The vertical arrow marks the time of the first $\bar{p}$ injection. The $\bar{p}$ sum signal before this arrow provides a check on the quality of the cancellation: it is typically 5 to 10 counts, well below the level from the true $\bar{p}$ signal, but above the noise of the system when no beam is in the machine, 1 to 3 counts.

The beam position, in $\mathrm{mm}$, is given by,

$$
P=26 \cdot \frac{|A|-|B|}{|A|+|B|}
$$

where the constant $26 . \mathrm{mm}$ is determined by the geometry of the pickups. While additional corrections are important for operation of the Tevatron, they would only complicate this paper and have been ignored. Figure 2b) shows the $p$ and $\bar{p}$ positions for the same time period as Figure 2a). The opening of the helix can be seen clearly in the $p$ trace.

There are no intentional changes to the central orbit during the opening of the helix and the $\bar{p}$ injection. Therefore one can predict the expected position of the $\bar{p}$ orbit at that time: it is the mirror image, about the central orbit, of the $p$ orbit. In Figure 2b) a dashed horizontal line is drawn at the predicted $\bar{p}$ position, obtained using the $p$ position immediately before and after the opening of the
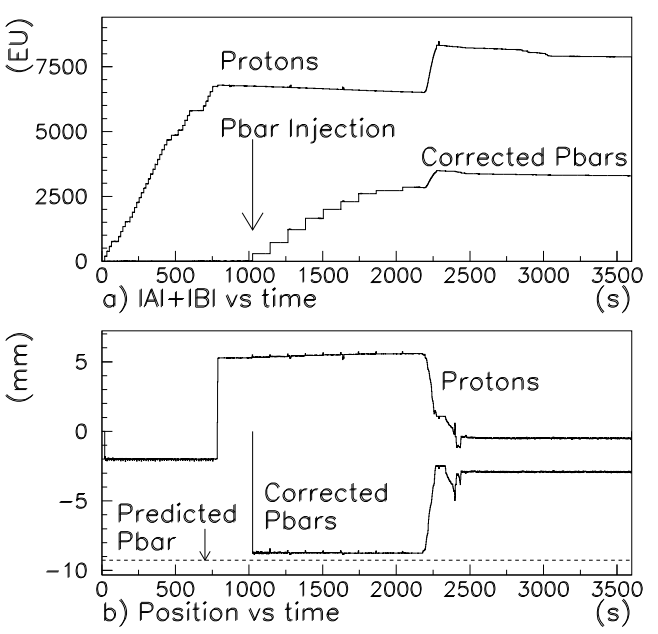

Figure 2: Sum signals and positions, after corrections, computed from the same shot as used in Figure 1.

helix. The measured $\bar{p}$ position agrees with the prediction to within $400 \mu \mathrm{m}$, which is within the accuracy specification of $1 \mathrm{~mm}$.

As the beam energy ramps up, near $2200 \mathrm{~s}$, the separator voltages are held constant. Therefore both beam species move at the same rate towards the central orbit. This is qualitatively observed in the data but the comparison is not exact because the central orbit does change during the ramp. After the ramp, the beams are squeezed and brought into collision, during which time there are large changes to the central orbit.

There is no measurable $\bar{p}$ position drift during the last five minutes of data from Figure $2 b$ ). The RMS width of the $\bar{p}$ position during this time was measured to be $27 \mu \mathrm{m}$, which includes the resolution of the BPM, the effect of incomplete cancellation of the proton contamination, and any true oscillations about the mean beam position. Turn by turn studies on the proton beam suggest that true beam motion is the dominant contribution. In any case, the RMS width is well within the resolution requirement of $<50 \mu \mathrm{m}$. Studies with other BPMs and other running conditions give RMS $\bar{p}$ widths in the range of 15 to $40 \mu \mathrm{m}$.

It was previously stated that the residual from the cancellation procedure is about 10 counts in the sum signal. Consider a worst case scenario in which all of the the excess is on one of the channels. Inspection of Figure 2a) and Equation 2 shows that, at full $\bar{p}$ current, this will result in a position bias of order $100 \mu \mathrm{m}$, well within the accuracy requirement.

Figure 3 shows a detail of Figure $2 \mathrm{~b}$ ) during $\bar{p}$ injection. This provides a another check on the quality of the cancellation of the $p$ contamination. When that cancellation is poor, the $\bar{p}$ position trace will show large steps at each $\bar{p}$ injection, as will be shown in Figure 5. In Figure 3 the $\bar{p}$ position is stable to better than $100 \mu \mathrm{m}$ throughout the $\bar{p}$ injection. The conclusion is that the cancellation is excellent.

Figure 3 also provides evidence for $\bar{p}$ contamination on 


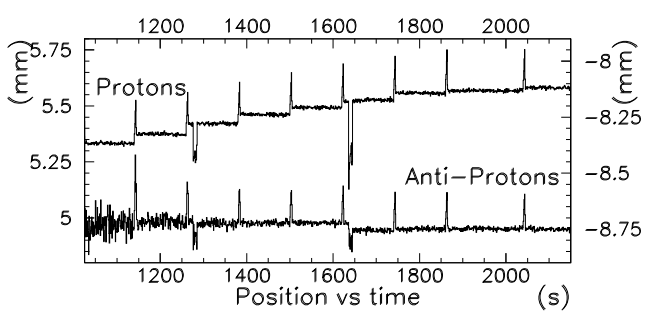

Figure 3: Detail of Figure 2b) during $\bar{p}$ injection. The left(right) scale is for the $p(\bar{p})$ position.

the $p$ position. The effect is about $300 \mu \mathrm{m}$, well below the accuracy specification of $1 \mathrm{~mm}$. It is understood in principle how to correct for this contamination but doing so would not result in significant operational improvements at the current $\bar{p}$ intensities. Moreover, calibrating this correction would probably require $\bar{p}$ only stores, a profligate use of $\bar{p}$ 's unless a significant operational improvement is promised. The injection bumps and the cogging operations are also clearly seen in Figure 3.

One of the Tevatron tune up steps is to inject a $p$ bunch and then energize the separators with the opposite polarity, which places the $p$ bunch on the $\bar{p}$ helix. Figure 4a) shows

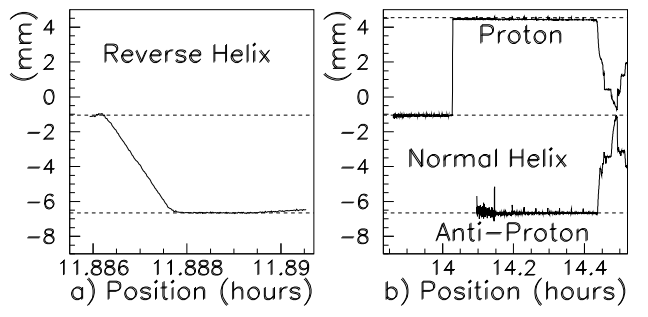

Figure 4: Comparison of $\bar{p}$ position with that of protons on the reversed helix, for BPM VA35. The time axis is time of day, in hours.

the measured $p$ position during one of these reverse helix stores. Horizontal dashed lines are drawn at the positions of the measured central and $\bar{p}$ orbits. Figure $4 \mathrm{~b}$ ) shows the measured beam positions for a shot which followed soon after. The two horizontal lines drawn on a) have been repeated in b). A third horizontal line has been drawn on b) at the predicted position of the $p$ helix. Inspection of the figure shows that the central orbit has moved by about $50 \mu \mathrm{m}$ between the two data sets. It also shows that the $\bar{p}$ 's are measured to be at the predicted position to better than $100 \mu \mathrm{m}$ and that the $p$ 's are at the mirror image position with an accuracy of about $150 \mu \mathrm{m}$. These deviations are within the specified tolerances.

In order to further test the self consistency of the upgraded BPM electronics, there is a plan for a $p$ only store with the separators off. During this store the measured $p$ position will trace out the central orbit from initial $p$ injection to the initiation of collisions. Immediately following this study, a normal physics shot will be done and the measured $p$ and $\bar{p}$ positions will be compared to the central orbit determined in the $p$ only store. If there are significant deviations from the expected mirror image model, a correction scheme will be developed.

The cancellation coefficients vary from one BPM to the next, presumably due to material and construction tolerances. The coefficients for a given BPM also vary from store to store. The source of this effect is believed to be changes in the vertical beam position at a horizontalmeasuring BPM, and vice versa. The scale of the store to store variation is illustrated in Figure 5, in which the upper points show the $\bar{p}$ position for a particular shot using the cancellation coefficients computed using the helix opening of that shot. The lower points show the positions for the same shot, but computed using the cancellation coefficients from a shot 7 days earlier. The older cancellation coeffi-

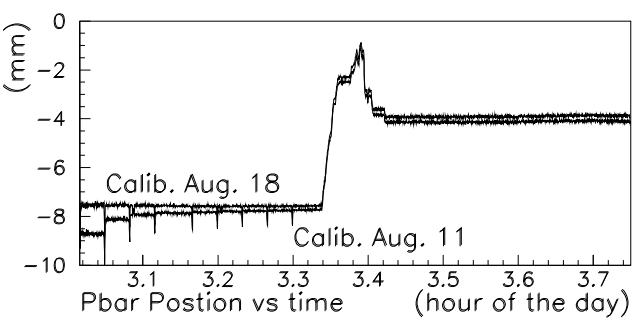

Figure 5: Time stability of the cancellation coefficients. See text for details.

cients do a much poorer job, particularly at low $\bar{p}$ currents, when the residual contamination is a much larger fraction of the total signal. When all $\bar{p}$ bunches have been injected, the bias from the stale calibration is about $500 \mu \mathrm{m}$, which is within requirements. When only a few bunches have been injected, however, the bias is outside of the requirements. To address this an automated procedure to recompute the cancellation coefficients every shot is being developed.

\section{CONCLUSIONS}

This paper has described the frequency domain method for measuring the $\bar{p}$ position using the upgraded Tevatron BPM system. The method has been shown to meet the requirements for resolution and accuracy, the accuracy tests being performed relative to the measured proton position.

\section{REFERENCES}

[1] R. E. Shafer,R. C. Webber, and T. H. Nicol, "Fermilab Energy Doubler Beam Position Dectector", IEEE NS-28, 3, (3390) June, 1981.

[2] R. C. Webber et al., "Using Time Separation of Signals to Obtain Independent Proton and Antiproton Beam Position Measurements Around the Tevatron", submitted to PAC05, May, 2005.

[3] S. Wolbers et al., "Tevatron Beam Position Monitor Upgrade", submitted to PAC05, May, 2005.

[4] E. James, G. Cancelo, and S. Wolbers, "Digital Signal Processing the Tevatron BPM Signals", submitted to PAC05, May, 2005. 